\title{
BRIEF REPORT \\ Risk Factors and Management of Deep Venous Thrombosis in Children Following Post-Surgical Hypopituitarism in Craniopharyngioma
}

\author{
Shanmuganathan Chandrakasan, MD, ${ }^{1}$ Sandeep Sood, $\mathrm{MD}^{2}$ Steven Ham, DO, ${ }^{2}$ Kathleen Moltz, MD, ${ }^{3}$ \\ Mary Jane Frey, $\mathrm{RN}^{1,4}$ and Madhvi Rajpurkar, $\mathrm{MD}^{1,4} *$
}

Post-operative hypopituitarism following craniopharyngioma (CP) surgery is treated by replacement of various hormones. The risk of deep venous thrombosis (DVT) following CP surgery and initiation of hormones has not been well studied and recognized. We present three patients with DVT who had family history of DVT and procoagulant risk factors such as inherited thrombophilia and elevated Von Willebrand factor levels due to treatment with desmopressin. We discuss the individualized management of anticoagulation and the dilemma of starting estrogen and progesterone replacement therapy in them. Pediatr Blood Cancer 2011;57:175177. (c) 2011 Wiley-Liss, Inc.

Key words: craniopharyngioma; deep venous thrombosis; hormonal replacement therapy; hypopituitarism; thrombophilia

\section{INTRODUCTION}

Craniopharyngioma $(\mathrm{CP})$ is the commonest suprasellar brain tumor and it constitutes $6-10 \%$ of pediatric brain tumors [1]. Although the tumor is slow growing and benign, managing long-term co-morbidities following surgery is challenging [2]. A common complication is the development of varying degrees of hypopituitarism (HP) which warrants long-term replacement of various hormones including estrogen and progesterone in girls at the onset of adolescence [3]. Deep venous thrombosis (DVT) has been occasionally reported in patients with $\mathrm{CP}$ [4-6]. In the immediate post-operative period multiple risk factors contribute to the development of DVT. Another potentially high risk period for DVT is during the initiation of estrogen and progesterone replacement therapy (HRT) in females with HP. Although the risk of DVT in association with HRT for post-menopausal women has been investigated, the risk for development of DVT in adolescent girls requiring HRT for HP has not been well studied. We report three female patients, who developed DVT after CP surgery, and highlight their unique risk factors for thrombosis, their management and discuss the need for an individualized approach to treatment.

\section{METHODS}

Our center has followed 34 patients with CP over the last 14 years, of which 3 patients developed DVT. All patients were investigated for thrombophilia (Table I) as per the recommendations of Perinatal and Pediatric Haemostasis Subcommittee of the Scientific and Standardization Committee of the International Society on Thrombosis and Haemostasis (ISTH) [7].

\section{RESULTS}

\section{Case 1}

A 16-year-old Caucasian female presented with left leg swelling after traveling for $6 \mathrm{hr}$. Ultrasound (US) showed non-visualization of the deep venous structures and venography confirmed extensive ileofemoral DVT. Her past medical history was significant for partial HP after resection of $\mathrm{CP}$ at 3 years of age. She was on thyroid replacement and 2 months prior to this event she was started on HRT with oral contraceptive pills (OCP) containing drospirenone $(3.0 \mathrm{mg})$ and ethinyl estradiol $(0.030 \mathrm{mg})$. Family history was significant for DVT in paternal aunt and cousin, both related to OCP use. Her OCP was stopped, and she was treated initially with therapeutic unfractionated heparin and later transitioned to enoxaparin for 6 months. Repeat imaging at the end of therapy showed complete resolution of thrombosis. Her thrombophilia work up showed that she was heterozygous for Factor V Leiden (FVL) and plasminogen activator inhibitor-1 (PAI-1) mutation $(4 \mathrm{G} / 5 \mathrm{G})$. After discussing the risk for recurrent thrombosis and the option of giving prophylactic anticoagulation therapy with HRT, the family elected to stop all HRT. Two years after stopping enoxaparin she has had no recurrence of DVT.

\section{Case 2}

A 13-year-old Caucasian female diagnosed with CP underwent total surgical resection. Following surgery, she developed panhypopituitarism and diabetes insipidus (DI) for which she was started on replacement therapy with hydrocortisone, synthroid, and intranasal desmopressin. Her immediate post-operative period was complicated by left sided hemiparesis due to right middle cerebral artery territory infarction. Two weeks later she developed extensive bilateral lower extremity DVT with extension into the inferior vena cava. A Gunther-Tulip inferior vena cava (IVC) filter was placed and she was subsequently treated with unfractionated heparin. She had strong family history of DVT in maternal grandmother, maternal great-grandmother, and maternal aunt; all of them were on coumadin. Thrombophilia work up revealed that she had elevated Von Willebrand factor Antigen (vWFAg) level of

\footnotetext{
${ }^{1}$ Carman and Ann Adams Department of Pediatrics, Children's Hospital of Michigan, Wayne State University, Detroit, Michigan; ${ }^{2}$ Division of Pediatric Neurosurgery, Children's Hospital of Michigan, Wayne State University, Detroit, Michigan; ${ }^{3}$ Division of Pediatric Endocrinology, Children's Hospital of Michigan, Wayne State University, Detroit, Michigan; ${ }^{4}$ Division of Pediatric Hematology Oncology, Children's Hospital of Michigan, Wayne State University, Detroit, Michigan
}

Conflict of interest: Nothing to declare.

*Correspondence to: Madhvi Rajpurkar, MD, Division of Hematology/Oncology, Children's Hospital of Michigan, 3901 Beaubien Blvd., Detroit, MI 48201.

E-mail: mrajpurk@med.wayne.edu

Received 19 July 2010; Accepted 2 November 2010 
TABLE I. Thrombophilia Testing for Patients With Deep Venous Thrombosis

\begin{tabular}{lll}
\hline & Thrombophilic trait & \multicolumn{1}{c}{ Method for testing } \\
\hline Level I & Factor V Leiden & PCR-RFLP \\
& Prothrombin G 20210A & PCR-RFLP \\
& Fasting homocysteine & FPIA \\
& Protein C, S, & Clotting or chromogenic \\
& antithrombin III & assay \\
& Lipoprotein (a) & NIPIA \\
& Antiphospholipid & Phospholipid based clotting \\
& antibodies & assays and anticardiolipin \\
& Factor VIII & One stage clotting assay \\
& vWF & Immunoassay \\
Level II & PAI-1 & PCR-RFLP \\
& MTHFR & PCR-RFLP \\
\hline
\end{tabular}

PCR-RFLP, polymerase chain reaction-restriction fragment length polymorphism; FPIA, fluorescence polarization immunoassay; NIPIA, near-infrared particle immunoassay; PAI-1, plasminogen activator inhibitor-1(4G/4G) polymorphism; MTHFR, methylenetetrahydrofolate reductase $677 \mathrm{C}>\mathrm{T}$ variant. ${ }^{\mathrm{a}}$ Tests based on clinical history and Level I results.

$403 \%$ (range 49-156\%), factor VIII (FVIII) activity of $418 \%$ (range $63-150 \%$ ) and PAI levels of $>100 \mathrm{IU} / \mathrm{ml}$ (range 0 $14 \mathrm{IU} / \mathrm{ml}$ ) and further genetic studies showed she was homozygous for PAI-1(4G/4G) polymorphism. She was transitioned to therapeutic enoxaparin for six months. During follow up she developed hypothalamaic hyperphagia leading to morbid obesity, type 2 diabetes mellitus, severe osteoporosis, narcolepsy, and seizures. Two years following the initial presentation, HRT was initiated. Since she had multiple risk factors for recurrence of DVT (Table II), prior to initiation of HRT she was started on prophylactic enoxaparin $80 \mathrm{mg}$ daily. Three years after the initiation of enoxaparin she continues to be on HRT without recurrence of DVT.

\section{Case 3}

A 5-year-old Caucasian female underwent surgical resection after diagnosis of CP. Following surgery, she developed panhypopituitarism and DI for which she was started on replacement therapy with hydrocortisone, synthroid, and intranasal desmopressin. Two months following surgery she was admitted with right leg swelling and altered sensorium. She was noted to have severe hypernatremic dehydration with serum sodium of $175 \mathrm{meq} / \mathrm{L}$. Doppler US of right leg showed extensive ileofemoral DVT. She was started on unfractionated heparin and later changed to therapeutic enoxaparin for a total duration of 3 months. Repeat imaging at 3 months showed resolution of DVT. She had a strong family history of thrombosis, paternal grandfather died of myocardial infarction at the age of 40 years and maternal uncle had DVT when he was 30 years of age. Thrombophilia screening revealed elevated vWFAg of $258 \%$ (range 49-156\%), FVIII of $204 \%$ (range $63-150 \%$ ), and the presence of homozygous 5,10methylenetetrahydrofolate reductase (MTHFR) $677 \mathrm{C}>\mathrm{T}$ variant with a normal serum homocysteine level. On follow-up, her DI is stable on desmopressin replacement. Due to her age, she has not required HRT currently. However, as thrombophilia screening has not revealed any known thrombosis predisposing mutations, our recommendation is to start low dose HRT without prophylactic anticoagulation with ongoing clinical monitoring for thrombosis.

\section{DISCUSSION}

Multiple co-morbidities following craniopharyingioma surgery predispose patients to the development of DVT. In the immediate post-operative period, prolonged immobilization with the release of procoagulant substance and the presence of central venous catheters are some of the known risk factors. Additionally, acquired risk factors that develop as a consequence of surgery predispose them to DVT. These include obesity and metabolic syndrome secondary to hypothalamic hyperphagia [8], desmopressin replacement therapy [4], and estrogen replacement therapy [9].

Both inadequate desmopressin replacement and desmopressin therapy has been reported to be associated with thrombosis $[4,10$ 12]. Furtado et al. [4] reported two cases of IVC thrombosis following $\mathrm{CP}$ surgery. The thrombosis was attributed to severe dehydration leading to hemoconcentration due to inadequate replacement of desmopressin [4]. In patients on desmopressin therapy, it has been reported to release vWF multimers and

TABLE II. Risk Factors and Management of DVT in Children With Craniopharyngioma

\begin{tabular}{|c|c|c|c|}
\hline & Patient 1 & Patient 2 & Patient 3 \\
\hline DVT & Yes & Yes & Yes \\
\hline Trigger & $\begin{array}{l}\text { OCP } \\
\text { Prolonged travel }\end{array}$ & Multiple & Hypernatremic dehydration \\
\hline Family history of DVT & Yes & Yes & Yes \\
\hline Ongoing risk & $\begin{array}{l}\text { Heterozygous-FVL } \\
\text { Heterozygous-PAI-1 } \\
\text { Obese }\end{array}$ & $\begin{array}{l}\text { High PAI-1 levels } \\
\text { Homozygous-PAI-1 } \\
\text { High vWFAg and factor VIII } \\
\text { Non-ambulatory } \\
\text { Metabolic syndrome }\end{array}$ & $\begin{array}{l}\text { Homozygous-MTHFR } \\
\text { High vWFAg and factor VIII }\end{array}$ \\
\hline Anticoagulation (enoxaprin) & Stopped & Continued $^{\mathrm{a}}$ & Stopped \\
\hline HRT & $\begin{array}{l}\text { No } \\
\text { Needs prophylactic anticoagulation } \\
\text { if HRT started in future }\end{array}$ & $\begin{array}{l}\text { Yes } \\
\text { On prophylactic anticoagulation } \\
\text { with no recurrence }\end{array}$ & $\begin{array}{l}\text { Yes } \\
\text { No prophylactic anticoagulation } \\
\text { as risk factors resolved }\end{array}$ \\
\hline
\end{tabular}

OCP, oral contraceptive pills; FVL, Factor V Leiden; PAI-1, plasminogen activator inhibitor-1(4G/4G) polymorphism; MTHFR, methylenetetrahydrofolate reductase $677 \mathrm{C}>\mathrm{T}$ variant. ${ }^{\mathrm{a}}$ Continued in prophylactic dose. 
transiently increase vWF level leading to increased risk of thrombosis [4,10-12]. High levels of vWF were seen in cases 2 and 3; case 3 also had associated severe dehydration. However, a large meta-analysis failed to show a statistically significant increase of thrombosis in patients who received desmopressin after surgery [13].

In adolescent females with HP HRT is recommended because estrogen and progesterone are required for initiation of puberty, fusion of epiphyses, breast development, menstruation, achievement of peak bone mass, and maintenance of normal adult female body composition [14]. One of the common means of estrogenprogesterone hormone replacement (HRT) is by OCP. However, its use is known to be associated with two- to sixfold increased risk of DVT [15]. Nevertheless, OCP containing low dose ethinyl estradiol $(0.020 \mathrm{mg})$ are associated with much lower risk [9]. Also, OCP that contain desogestrel or gestodene (third generation progesterone) seem to be associated with greater risk than those that contain levonorgestrel (second generation progesterone) [9]. These factors should be taken into consideration before initiation of HRT in these patients. Of increasing interest is the use of the more physiological transdermal estrogen patch for HRT. Although the risk of developing DVT is similar to standard dose estrogen containing OCP [16] but when compared to OCP it has the added advantage of decreased requirement of growth hormone replacement for HP [14].

The risk of DVT increases with the concomitant presence of associated thrombophilia. All of our three patients had a family history of thrombosis. Thrombophilic states were identified in two of our patients, which include; heterozygous FVL and elevated PAI activity with PAI-1 4G/5G polymorphism in case 1 , markedly increased PAI activity with $4 \mathrm{G} / 4 \mathrm{G}$ homozygous polymorphism in case 2 . In case 3 , the thermolabile MTHFR mutation was identified but the homocysteine level was normal. Of all the potential procoagulant states identified, the risk of DVT with FVL mutation in patients on HRT has been well studied. Patients with heterozygous FVL have a five- to tenfold increased risk of DVT when initiated on OCP [17]. It is controversial whether the PAI $4 \mathrm{G} / 4 \mathrm{G}$ polymorphism is a true thrombophilic mutation. Some reports have shown such an association while others have refuted this $[18,19]$. The MTHFR C677T mutation with hyperhomocysteinemia is associated with increased risk of thrombosis, but its significance in the absence of elevated homocysteine is not well established [20].

In conclusion, children with CP develop multiple co-morbidities that increase the risk for DVT. A detailed family history and individualized screening for thrombophilia may be beneficial in prevention and management of DVT and HRT.

\section{REFERENCES}

1. Sanford RA, Muhlbauer MS. Craniopharyngioma in children. Neurol Clin 1991;9:453-465.

2. Gleeson H, Amin R, Maghnie M. 'Do no harm': Management of craniopharyngioma. Eur J Endocrinol 2008;15:S95-S99.

3. Kalina MA, Skala-Zamorowska E, Kalina-Faska B, et al. Practical approach to childhood craniopharyngioma: A role of an endocrinologist and a general paediatrician. Childs Nerv Syst 2009;25:1053-1060.

4. Furtado SV, Dash PK, Reddy K, et al. Budd-Chiari syndrome, ascites and shunt malfunction due to hyperosmolar hypernatremia in operated pediatric craniopharyngiomas: A red herring. Childs Nerv Syst 2008;24:1051-1055.

5. Takamura Y, Uede T, Hashi K, et al. A case of pulmonary embolism with diabetes insipidus developed after removal of craniopharyngioma. No Shinkei Geka 1990;18:199-203.

6. Sekaran VI, Muthu SS, Velu SK, et al. Craniopharyngioma presenting as cavernous sinus thrombosis. J Indian Med Assoc 1998;96:285-287.

7. Manco-Johnson MJ, Grabowski EF, Hellgreen M, et al. Laboratory testing for thrombophilia in pediatric patients. On behalf of the Subcommittee for Perinatal and Pediatric Thrombosis of the Scientific and Standardization Committee of the International Society of Thrombosis and Haemostasis (ISTH). Thromb Haemost 2002;88:155-156.

8. Eichinger S, Hron G, Bialonczyk C, et al. Overweight, obesity, and the risk of recurrent venous thromboembolism. Arch Intern Med 2008;168:1678-1683.

9. Lidegaard O, Lokkegaard E, Svendsen AL, et al. Hormonal contraception and risk of venous thromboembolism: National follow-up study. BMJ 2009;339:b2890.

10. Lowe GD. Desmopressin and myocardial infarction. Lancet 1989;1:895-896.

11. McLeod BC. Myocardial infarction in a blood donor after administration of desmopressin. Lancet 1990;336:1137-1138.

12. Mannucci PM, Carlsson S, Harris AS. Desmopressin, surgery and thrombosis. Thromb Haemost 1994;71:154-155.

13. Crescenzi G, Landoni G, Biondi-Zoccai G, et al. Desmopressin reduces transfusion needs after surgery: A meta-analysis of randomized clinical trials. Anesthesiology 2008;109:1063-1076.

14. Mah PM, Webster J, Jonsson P, et al. Estrogen replacement in women of fertile years with hypopituitarism. J Clin Endocrinol Metab 2005;90:5964-5969.

15. Gomes MP, Deitcher SR. Risk of venous thromboembolic disease associated with hormonal contraceptives and hormone replacement therapy: A clinical review. Arch Intern Med 2004; 164:1965-1976.

16. Jick S, Kaye JA, Li L, et al. Further results on the risk of nonfatal venous thromboembolism in users of the contraceptive transdermal patch compared to users of oral contraceptives containing norgestimate and 35 microg of ethinyl estradiol. Contraception 2007;76:4-7.

17. Spannagl M, Heinemann LA, Schramm W. Are factor V Leiden carriers who use oral contraceptives at extreme risk for venous thromboembolism? Eur J Contracept Reprod Health Care 2000; $5: 105-112$.

18. Balta G, Altay C, Gurgey A. PAI-1 gene 4G/5G genotype: A risk factor for thrombosis in vessels of internal organs. Am J Hematol 2002;71:89-93.

19. Segui R, Estelles A, Mira Y, et al. PAI-1 promoter 4G/5G genotype as an additional risk factor for venous thrombosis in subjects with genetic thrombophilic defects. Br J Haematol 2000; 111:122-128.

20. Naess IA, Christiansen SC, Romundstad PR, et al. Prospective study of homocysteine and MTHFR 677TT genotype and risk for venous thrombosis in a general population-results from the HUNT 2 study. Br J Haematol 2008;141:529-535. 